\title{
Corela
}

Cognition, représentation, langage

$11-2 \mid 2013$

Vol. $11, \mathrm{n}^{\circ} 2$

\section{Les représentations cognitives : pour une mise en évidence de leur rôle dans l'appropriation des langues}

Cognitive representations and their possible role in language learning

\author{
Laurence Vincent-Durroux
}

\section{(2) OpenEdition}

Journals

Édition électronique

URL : http://journals.openedition.org/corela/2983

DOI : $10.4000 /$ corela.2983

ISSN : 1638-573X

Éditeur

Cercle linguistique du Centre et de l'Ouest - CerLICO

Référence électronique

Laurence Vincent-Durroux, « Les représentations cognitives : pour une mise en évidence de leur rôle dans l'appropriation des langues », Corela [En ligne], 11-2 | 2013, mis en ligne le 17 février 2014, consulté le 08 mai 2019. URL : http://journals.openedition.org/corela/2983 ; DOI : 10.4000/ corela.2983

Ce document a été généré automatiquement le 8 mai 2019.

\section{(c) (i) (2)}

Corela - cognition, représentation, langage est mis à disposition selon les termes de la licence Creative Commons Attribution - Pas d'Utilisation Commerciale - Partage dans les Mêmes Conditions 4.0 International. 


\title{
Les représentations cognitives : pour une mise en évidence de leur rôle dans l'appropriation des langues ${ }^{1}$
}

\author{
Cognitive representations and their possible role in language learning
}

\author{
Laurence Vincent-Durroux
}

1 Les effets de la langue maternelle sur l'appropriation de la langue seconde font l'objet d'analyses parfois contradictoires. Cela pourrait-il provenir du fait que certains éléments constitutifs des langues jouent un rôle encore mal connu et que sont notamment sousestimés les conflits entre les représentations cognitives sous-jacentes à chaque langue lors de l'apprentissage d'une langue seconde ? Cet article a pour objectif de mettre en évidence le principe d'existence de ces conflits et le fait que les représentations cognitives construites antérieurement jouent un rôle limitatif lors de l'apprentissage ultérieur des langues. Ce principe pourrait contribuer à expliquer les phénomènes de fossilisation et de nativisation en langue seconde, phénomènes décrits notamment par Selinker \& Lamendella (1980), Selinker \& Lakshamanan (1992), Andersen (1983) et NarcyCombes (2005).

2 Nous montrons donc ci-dessous le rôle limitatif des représentations cognitives initiales en examinant un contexte dans lequel le développement cognitif précède l'appropriation linguistique: ce contexte est celui de l'oralisme en cas de surdité profonde prélinguistique. Tenus à l'écart des langues signées, les petits sourds accèdent à leur première langue (la langue maternelle orale) de manière différée par rapport aux petits entendants. Leurs productions langagières à l'adolescence comportent en effet des particularités qu'il est possible de rattacher à des conceptions qui leur sont spécifiques, montrant l'influence du cognitif sur le linguistique. L'étude de ce contexte permet de proposer une généralisation puisque le point de départ où peuvent être observées les représentations cognitives n'est pas lié à une langue particulière. 
3 Nous rappelons tout d'abord quelques liens entre la langue maternelle et les langues secondes, puis décrivons les relations possibles entre les représentations cognitives et les langues avant d'aborder le contexte de l'oralisme et son apport à notre problématique.

\section{Liens envisageables entre la langue maternelle et les langues apprises ultérieurement}

4 Dans l'apprentissage des langues, la langue maternelle (LM) peut être prise en compte de plusieurs manières. Il s'agit tout d'abord du point de référence par rapport auquel un jugement de différence peut être posé, jugement qui fait considérer comme « étrangères " les langues autres que la langue maternelle. De quel ordre ce point de référence est-il, plus précisément, dans la construction des langues acquises ultérieurement?

5 Le rôle de la LM est évoqué de manière contradictoire dans les étapes intermédiaires de l'apprentissage d'une langue seconde (LS), étapes qualifiées d'interlangue. Dans cette étape, les formes linguistiques observées ne sont, semble-t-il, ni celles de la LM ni celles de la LS. Certains auteurs considèrent qu'il y a une influence de la LM et de la LS sur l'interlangue (Hendriks et al., 2008; Lambert et al., 2008 ; Perdue, 1993 ; Slobin, 2004). Mais d'autres chercheurs, avec des populations d'apprenants de LM différentes, observent des erreurs communes, ce qui les conduit à considérer que ces erreurs sont liées à la LS et sont indépendantes de la LM (Dulay \& Burt, 1973 ; 1974).

6 Un autre lien entre LM et LS concerne les processus d'apprentissage, qui pourraient être communs puisque l'apprenant de LS emploie des stratégies d'apprentissage qui trouvent leur source dans le développement de sa LM (Lenart, 2006; Watorek, 2004). De cette manière, la LM est un point d'appui nécessaire pour que l'apprenant parvienne aux étapes de l'interlangue. Ces stratégies d'apprentissage sont des stratégies cognitives telles que la sur-généralisation et la simplification qui ne sont pas nécessairement spécifiques à l'apprentissage des langues.

7 Enfin, le niveau de maîtrise des locuteurs natifs dans leur LM peut-il être atteint par un apprenant en LS ? Les linguistes sont partagés, notamment lorsque l'apprentissage de la LS n'a pas débuté dès le plus jeune âge; majoritairement, les études montrent que l'apprenant ne parvient qu'exceptionnellement à atteindre en LS le niveau du locuteur natif (Birdsong, 2007). La présence de la LM pourrait être mise en cause dans cette réalité ; de quelle manière toutefois?

\section{Langues, représentation du monde, cognition}

8 Les théories cognitives établissent des rapports entre les perceptions que l'être humain a du monde et les conceptions qui en découlent et sont observables dans la langue :

Chaque langue découpe le réel à son gré, organise le pensable, génère une interprétation du monde. (... ) La complexité du réel n'autorise pas la pensée à la dominer dans son entier: celle-ci n'en saisit jamais que des facettes, qu'elle réorganise au mieux de ses besoins. (Martin, 1998 : 40-41)

9 La langue unifierait une communauté cognitive partageant un réseau de représentations (Donald, 2001, cité par Lapaire, 2006: 16). Le terme de "représentations" peut être compris comme une reconstruction de la réalité perçue, selon la métaphore de la diplomatie qui opère la distinction entre la représentation et ce qui est représenté 
(Havelange et al., 2002: 115). En linguistique, la notion de « représentations » trouve son origine dans la théorie de Guillaume pour qui « le langage humain n'existe qu'à partir du moment où le vécu expérimental est muté en représentation » (Guillaume, 1990 : 179).

10 Selon sa langue maternelle, le sujet peut être amené à sélectionner des informations différentes dans l'extralinguistique. Concernant l'espace par exemple, il apparaît qu'une schématisation, et donc une simplification, sont effectuées : certains aspects de la scène de référence sont sélectionnés au détriment d'autres (Talmy, 1988 ; 2000, vol. 1 : 177). Par exemple, le locuteur francophone focalise davantage dans son discours l'indication de la direction du déplacement que celle du mode de déplacement ("Il est revenu en voiture »), contrairement au locuteur anglophone («He drove back»). Les formes de la langue sont mises au service de la focalisation retenue (ibid.) : le français est une langue à cadrage verbal (l'indication de direction est intégrée au verbe : revenir) alors que l'anglais est une langue à cadrage de satellite (l'indication de direction est extérieure au verbe : back). En plus des structures syntaxiques et des éléments morphologiques, phonologiques et lexicaux qui lui sont propres, toute langue serait caractérisée par des représentations cognitives associées. L'apprenant entrerait donc dans la LS muni des représentations cognitives conférées par sa LM.

11 Ces représentations cognitives interfèrent-elles avec celles qui sont propres à la languecible? Pour démontrer que les représentations cognitives jouent effectivement un rôle dans l'influence de la LM sur l'apprentissage de la LS, il faudrait pouvoir dissocier les représentations cognitives sous-jacentes et les éléments de surface de la LM (phonologie, morpho-syntaxe, lexique). Une telle entreprise est complexe, voire impossible. En revanche, le principe potentiellement à l'œuvre, c'est-à-dire l'influence des représentations cognitives initiales sur la langue acquise ultérieurement, peut être mis en évidence dans le contexte de l'oralisme en cas de surdité profonde prélinguistique. En effet, dans ce contexte, il est possible d'isoler chronologiquement la construction des représentations cognitives et celle de la langue: le jeune sourd profond peut vivre plusieurs mois ou années sans accès à une langue tout en développant des représentations cognitives par ses perceptions sensorielles.

\section{Surdité prélinguistique et représentations cognitives}

12 La surdité profonde peut être acquise au cours de la vie ou bien être congénitale. Depuis 1988 , nous menons des travaux de recherche sur la langue orale de jeunes sourds oralistes atteints d'une surdité congénitale et prélinguistique. Chez ces sujets, le choix des parents a été de les amener à utiliser la langue de leur communauté entendante comme moyen de communication, sans langue des signes. La langue orale est dans ce cas la langue maternelle de l'enfant, mais contrairement aux enfants entendants, l'accès à cette langue est différé pour les jeunes sourds profonds.

13 La langue orale n'est pas la modalité de communication naturelle des sourds profonds puisque l'oral s'appuie sur un canal sensoriel, l'audition, que la personne sourde n'a pas à sa disposition. Pour que la langue orale puisse tout de même être développée en contexte de surdité profonde, il y a donc des préalables :

14 - la surdité doit avoir été dépistée ; ce dépistage intervient désormais de plus en plus tôt, souvent au cours de la première année de vie. Des examens peuvent être faits à la 
naissance mais leurs résultats nécessitent d'être confirmés par un diagnostic effectué entre 18 et 24 mois ; des entendants. Cela entraîne-t-il une spécificité lors de l'appropriation de la langue maternelle, appropriation différée par rapport aux enfants entendants?

\section{Les langues orales des sourds ou « langues sourdes »}

20 Dans les langues, certains phénomènes peuvent être reliés aux représentations cognitives du spatio-temporel : pour l'espace, il s'agit en particulier de la détermination nominale, des prépositions et de la place des référents dans l'espace énonciatif. Pour le temps, il s'agit notamment des temps verbaux. Nous présentons ici plus particulièrement la détermination nominale, les prépositions et les indications temporelles, telles que les sourds se les approprient dans les langues-cibles que sont l'anglais ou le français. Les 
observations proviennent de l'analyse de nos données (environ 4000 énoncés oraux) recueillies auprès de vingt sourds profonds, francophones ou anglophones, âgés de 10 à 14 ans. Tous les sujets présentaient une surdité bilatérale et prélinguistique. Ils étaient scolarisés en intégration et n'avaient pas utilisé de langues des signes. Le protocole d'enregistrement a permis de solliciter des discours de différents types, donnant lieu à une variété de formes: discours narratifs, descriptifs, argumentatifs, avec ou sans support. Les données ont fait l'objet d'une transcription orthographique puis d'analyses quantitatives et qualitatives (Vincent-Durroux, à paraître en 2014).

\subsection{La détermination nominale}

21 La détermination nominale permet de délimiter le domaine notionnel (structure mentale dans laquelle sont organisées les occurrences des représentations mentales, les notions) et de construire des valeurs référentielles par projection du linguistique sur l'extralinguistique. L'espace est donc particulièrement impliqué dans ces opérations. Il ressort de nos premières données (1988) que les déterminants sont inadéquats vis-à-vis de la langue de référence dans $25 \%$ des cas, et que la fonction du groupe nominal dans l'énoncé est décisive : en fonction sujet, le groupe nominal reçoit majoritairement une détermination adaptée alors que ce n'est pas le cas en position de complément d'objet ou de groupe prépositionnel (Vincent-Durroux, 2009:5) : le bébé prend petite pelle; and a cow was reading newspaper; I went to canoe trip for weekend.

Lorsque la forme de détermination se démarque de celles observées dans la langue-cible, la dissociation des valeurs quantitative et qualitative est impliquée tant chez les sourds anglophones que chez les sourds francophones. Le déterminant $\varnothing$ (valeur qualitative) est fréquent mais il est presque toujours associé à des termes variés précisant l'occurrence dans l'espace, termes relevant du pôle quantitatif. Ces termes isolent certaines propriétés des occurrences : elles analysent le référent, comme cela est décrit par ailleurs pour les adjectifs (Cotte, 1996 : 134), et permettent de le désigner en tant qu'occurrence distincte de toutes les autres. Ces expressions assurent une identification des référents par leurs relations fonctionnelles ou par ce qui assure leur homogénéité, comme leur couleur ou leur matière (Vincent-Durroux, 1999: 105). Ces termes peuvent être des adjectifs (il caresse ours brun; it is blue bedroom), des propositions subordonnées relatives (il y a hippopotame qui a déguisé en marron et rose) ou encore des groupes prépositionnels indicateurs de fonction (my mother's desk is desk for homework), de localisation (dans les arbres, fleurs quand même) ou de destination (tout près de salle de bains pour mon parent). Dans les groupes prépositionnels, les prépositions concernées sont surtout celles qui évoquent le lieu ou le temps : SUR, À COTÉ DE, PRÈS DE, DANS pour le français et ON, FOR, AT, TO, IN, AFTER, BESIDE pour l'anglais.

Cette spécificité des sourds pour la construction des occurrences met en évidence un ancrage particulier dans l'espace et le temps des situations d'énonciation.

\subsection{Les prépositions}

Pour la plupart, les prépositions trouvent leur origine dans la représentation de l'espace (Groussier, 1997 : 222). De nombreux cas d'emplois problématiques sont relevés dans nos données, tant chez les sourds anglophones que chez les sourds francophones, et ils sont de trois ordres: des prépositions superflues (le mur elle avait avec un miroir), des 
prépositions non adaptées au contexte (après il va dans la caissière), des prépositions absentes (puis il joue une poupée). Les deux derniers cas sont les plus fréquents. Que révèlent-ils? langues des signes et se sont attribué les langues orales de leur communauté, avec les particularités décrites ci-dessus. D'autres personnes sourdes utilisent les langues signées, dont les formes émanent des sourds eux-mêmes. Nous avons cherché à savoir si des correspondances existent entre les particularités identifiées dans les productions recueillies pour notre étude et les usages des langues signées, pour prendre en compte l'influence de la surdité sur la langue, indépendamment de sa modalité. Nous avons ainsi pu mettre en évidence le fait qu'en langue des signes belge, les prépositions absentes dans nos corpus n'auraient pas correspondu à un signe explicite dans 50 cas sur 55 (VincentDurroux, 2008: 13). Il apparaît ainsi que tous les sujets sourds mettent en œuvre les prépositions selon des critères qui leur sont propres et qui sont fortement liés à des spécificités d'ordre spatio-temporel.

\subsection{Les indications temporelles}

Les indications temporelles présentent elles aussi des particularités chez les sourds. Dans les tâches descriptives, les sourds emploient de manière récurrente l'imparfait et le prétérit. La valeur de rupture temporelle et de détachement entre la situation de référence et la situation d'énonciation, qui est associée à ces temps chez les entendants, 
semble être élargie afin d'indiquer une autre rupture temporelle : le référent de la tâche (les images) préexiste à la tâche de description elle-même. représentations cognitives en rapport avec la LM interfèrent avec la LS dont les conceptions cognitives sont autres. Cela pourrait avoir un effet de blocage qui expliquerait l'existence d'un plafond dans le développement de la langue-cible, dans l'apprentissage d'une LS (Birdsong, 2007) tout autant que chez les sourds (Bochner \& Albertini, 1988 : 28-29). L'apprenant ne parviendrait jamais totalement à faire abstraction de ses représentations cognitives initiales pour s'approprier celles qui sont sous-jacentes à la langue-cible.

En rapport avec ce plafond, des phénomènes de fossilisation (Selinker \& Lamendella, 1980 ; Selinker \& Lakshamanan, 1992) sont observés : cela signifie que malgré un niveau très avancé dans l'apprentissage, l'apprenant continue à faire systématiquement une ou des erreurs sur un point particulier de la LS. Ces zones de fragilité sont décrites pour le français langue étrangère (LE), l'anglais LE, l'italien LE, l'allemand LE, l'espagnol LE chez des locuteurs de langues maternelles aussi variées que l'anglais, le néerlandais, le finnois, le suédois, le français, le danois, l'italien, l'allemand, l'espagnol (Bartning, 1997). Les phénomènes de fragilité sont observés dans l'emploi des déterminants, de l'aspect, du temps ainsi que du lexique. La fragilité est donc de grande ampleur puisqu'elle s'observe dans de multiples catégories linguistiques ainsi que pour des situations interlinguistiques fort variées. Une telle ampleur permet d'envisager que l'obstacle ne provienne pas nécessairement des formes linguistiques de surface mais que les représentations cognitives qui les sous-tendent puissent être incriminées. 
Enfin, la nativisation est un phénomène récurrent, décrit en didactique des langues (Andersen, 1983; Narcy-Combes, 2005) et par lequel l'apprenant affecte à la LS des représentations erronées importées de la LM en traitant les nouvelles données de la LS selon des critères propres à la LM. Ce phénomène est difficile à prendre en compte dans l'enseignement des langues. Lorsque l'exposition à la LS est insuffisante pour que l'usage puisse contrecarrer ces phénomènes, il peut être efficace de proposer à l'apprenant une démarche métalinguistique et contrastive entre LM et LS pour lui permettre d'enclencher les processus de "dénativisation», "dénativisation qui permettra d'effacer les représentations erronées et de les remplacer par celles qui correspondent bien au système de la L2» (Demaizière \& Narcy-Combes, 2005). De même, le recours au mouvement et au spatio-temporel chez l'apprenant pourrait contribuer à «stimuler les opérations abstraites de conceptualisation » (Lapaire, $2006: 101)$ propres à la LS et à en faciliter l'apprentissage.

\section{Conclusion}

Etudier le contexte linguistique de la surdité présente en soi un grand intérêt pour mieux comprendre comment l'enfant sourd accède au langage malgré l'obstacle majeur qu'est l'absence d'audition. Mais, de même que les études sur l'aphasie peuvent révéler certains pans de la langue et du langage, les études sur la surdité permettent de mettre en évidence des phénomènes difficilement observables chez les locuteurs qui ne sont pas porteurs d'une atteinte sensorielle. Le fait qu'il y ait, chez les jeunes sourds oralistes, une dissociation ponctuelle du développement cognitif et du développement linguistique, permet d'apporter des arguments solides sur l'existence de conflits dus aux représentations cognitives en contexte d'apprentissage des langues secondes par des personnes entendantes.

\section{BIBLIOGRAPHIE}

\section{Références bibliographiques}

ANDERSEN M. (1983). "Pidginization and creolization in language acquisition". New York : Newbury House.

BARTNING I. (1997). "L'apprenant dit avancé et son acquisition d'une langue étrangère", Acquisition et interaction en langue étrangère [En ligne], 9 | 1997, mis en ligne le 18 novembre 2011. http://aile.revues.org/1316

BIRDSONG D. (2007). "Native-like pronunciation among late learners of French as a second language". In Language Experience in Second Language Speech Learning. Bohn O.S. \& Munro M. (dir.). Amsterdam : John Benjamins, 99-116. 
BOCHNER J.H \& ALBERTINI J.A. (1988). "Language varieties in the deaf population and their acquisition by deaf children and adults". In Language Learning and Deafness. Strong M. (dir.). Cambridge : Cambridge University Press, 3-48.

CADIOT P. (1997). Les prépositions abstraites en français, Paris, Armand Colin.

COTTE P. (1996). L'explication grammaticale de textes anglais, Paris : Presses Universitaires de France. DEMAIZIÈRE F. \& NARCY-COMBES J.-P. (2005). "Méthodologie de la recherche didactique : nativisation, tâches et TIC". Apprentissage des Langues et Systèmes d'Information et de Communication (Alsic), n 8 (1), 45-64. http://alsic.revues.org/index326.html

Dulay H. \& BuRT M. (1973). "Should we teach children syntax?" Language Learning, 23, 95-123.

Dulay H. \& BURT M. (1974). "Natural sequences in child second language acquisition". Language Learning, 24, 37-53.

GROUSSIER M.-L. (1997). "Prépositions et primarité du spatial : de l'expression de relations dans l'espace à l'expression de relations non-spatiales", Faits de langues, $\mathrm{n}^{\circ}$ 9, 221-234.

GUILLAUME G. (1990). Leçons de linguistique 1956-1957. Lille : Presses Universitaires de Lille.

HAVELANGE V., LENAY C. et STEWART J., "Les représentations mémoire externe et objets techniques", Intellectica, $\mathrm{n}^{\circ} 2,2002$, p. 115-119.

HendRiks H., Hickmann, M. \& Demagny A.-C. (2008). "How adult English learners of French express caused motion: a comparison with English and French natives". AILE, 27 : 15-41.

JIMENEZ M. (1997). La psychologie de la perception. Paris : Flammarion, collection Dominos.

LAFON J.-C. (1985). Les enfants déficients auditifs. Villeurbanne : SIMEP.

LAmbert M., CARroll M., von Stutterheim C. (2008). "Acquisition en L2 des principes d'organisation de récits spécifiques aux langues". AILE, 26 : 11-29.

LAPAIRE J.-R. (2006). La grammaire anglaise en mouvement. Paris : Hachette Education.

LENART E. (2006). Acquisition des procédures de détermination nominale dans le récit en français et polonais L1, et en français L2. Étude comparative de deux types d'apprenant : enfant et adulte. SaintDenis : Université Paris 8 . Thèse de doctorat.

MARTIN R. (1998). "Sur la distinction du signifié et du concept". In Leeman D. \& Boone A. et alii (dir.), Du percevoir au dire - Hommage à André Joly. Paris : L'Harmattan, 37-53.

MORGENSTERN A. \& SEKALI M. (2009). "La naissance d'une catégorie : étude contrastive de l'émergence des prépositions chez l'enfant en anglais et en français". In : François J., Gilbert É., Guimier C. \& Krause M. (dir.), Autour de la préposition, Caen : Presses Universitaires de Caen, "Bibliothèque de syntaxe et sémantique", 317-328.

NARCY-COMBES J.-P. (2005). Didactique des langues et TIC. Paris : Ophrys.

PERDUE C. (dir.) (1993). Adult language acquisition: cross-linguistic perspectives. Cambridge : Cambridge University Press.

SelinKer L., \& LAmendella J. T. (1980). "Fossilization in interlanguage learning". In Reading on English as a second language. Croft K. (dir.). Boston, MA : Little, Brown and Company, 132-143.

SelinKer L., \& LAKSHAMANAN U. (1992). "Language transfer and fossilization: The 'Multiple Effects Principle'". In Language transfer in language learning. Gass S. M \& Selinker L. (dir.), Amsterdam : John Benjamins, 197-216. 
SLOBIN D. I. (2004). "The many ways to search for a frog: Linguistic typology and the expression of motion events." In Relating events in narrative: Vol. 2. Typological and contextual perspectives. Strömqvist S. \& Verhoeven L. (dir.). Mahwah, NJ : Lawrence Erlbaum Associates, 219-257.

TALMY L. (1988). "The relation of grammar to cognition". In Topics in Cognitive Linguistics. RudzkaOstyn B. (dir.). Amsterdam : Benjamins, 165-205.

TALMY L. (2000). Toward a cognitive semantics. Volume 1: Concept structuring systems. Volume 2: Typology and process in concept structuring. Cambridge (MA) : MIT Press.

VINTER S. (2000). "Temps - langage - audition". Rééducation orthophonique, juin 2000, 202, 55-68.

VINCENT-DURROUX L. (1999). "Référenciation et opérations d'individuation dans la langue orale de sourds profonds anglophones et francophones". CYCNOS, volume 16, $\mathrm{n}^{\circ} 2,99-110$.

VINCENT-DURRoux L. (2008). "Prépositions et langage chez de jeunes sourds profonds oralistes". Corela (Cognition, représentation, langage), vol. $6, \mathrm{n}^{\circ} 1$. Accessible en ligne à l'URL : http://edel.univpoitiers.fr/corela/document.php?id=1868

VINCENT-DURRoux L. (2009). "Deaf Languages: Does the Hypothesis Still Apply?". Corela (Cognition, représentation, langage), vol. 7, $\mathrm{n}^{\circ} 2$. Accessible en ligne à l'URL : http://edel.univ-poitiers.fr/ corela/document.php?id=2244

VINCENT-DURROUX L. (à paraître en 2014). La langue orale des jeunes sourds. Paris : de Boeck- Solal, collection Voix, Parole, Langage.

WATOREK M. (2004). "Construction du discours par des enfants et des apprenants adultes". Langages, 155, 3-13.

\section{NOTES}

1. Que soient remerciés le Professeur Jean-Marie Merle (Université de Nice Sophia Antipolis) pour avoir inspiré certains aspects de ce texte, ainsi que tous les jeunes sourds, leurs parents et les soignants qui ont rendu possible cette étude.

\section{RÉSUMÉS}

De nombreuses études sur l'apprentissage des langues étrangères montrent que l'apprenant est incapable d'atteindre le niveau d'un locuteur natif. Des phénomènes de fossilisation et de nativisation demeurent, même à un niveau très avancé (Selinker \& Lamendella, 1980 ; Selinker \& Lakshamanan, 1992 ; Andersen, 1983 ; Narcy-Combes, 2005). Cet article met en évidence le rôle limitatif des représentations cognitives chez l'apprenant dans un contexte où il est possible d'isoler chronologiquement la construction des représentations cognitives et celle d'une première langue. Ce contexte est celui de la surdité profonde prélinguistique, et plus spécifiquement celui de l'oralisme : l'acquisition de la langue y est différée de quelques mois ou années sans qu'il n'y ait de système linguistique préalable puisque les oralistes sont tenus à l'écart des langues signées. Les jeunes sourds développent des représentations cognitives dans 
leurs premières années de vie, préalablement à l'accès à une première langue. Or, des études expérimentales (ex. Vinter, 2000) montrent que les sujets sourds ont une approche inhabituelle du spatial et du temporel, approche pouvant être mise en rapport avec l'absence de perception auditive. Du fait des liens entre perception sensorielle et conception (ex. Talmy, 1988 \& 2000), les représentations cognitives des sujets sourds relativement à l'espace et au temps seraient donc spécifiques. La langue orale produite par ces sujets à l'adolescence présente quant à elle des caractéristiques inhabituelles dans les domaines de la langue qui sont liés à l'espace et au temps, et ce, aussi bien chez les anglophones que chez les francophones dont les productions orales ont été analysées (ex. Vincent-Durroux, 2009 \& 2008). Les représentations cognitives influent donc sur l'appropriation de la langue par les sourds. La discussion porte sur le fait que, dans le contexte de l'apprentissage de langues étrangères par des entendants, les représentations cognitives sous-jacentes à la langue maternelle pourraient avoir un rôle limitatif et ainsi expliquer la persistance des phénomènes de fossilisation et de nativisation.

Some studies on second language learning show that learners cannot reach the level of native speakers. Even at an advanced stage, learners' productions can be characterized by phenomena known as "fossilisation" and "nativisation" (Selinker \& Lamendella, 1980; Selinker \& Lakshamanan, 1992; Andersen, 1983; Narcy-Combes, 2005). In this paper, the restrictive role of cognitive representations is established in a context where the construction of cognitive representations and the development of the first language are chronologically isolated: the context of the oral deaf with prelinguistic profound deafness. In that context, the development of cognitive representations clearly precedes that of a language, since the oral deaf are kept away from sign languages. Experimental studies (e.g. Vinter, 2000) show that deaf children's conceptions of space and time differ from those of hearing children, a result which could be related to the lack of aural perception. The links between sensory perception and conceptions (e.g. Talmy, 1988 \& 2000) could be implied, leading to specific space and time conceptions in the deaf. The analysis of oral data gathered from oral deaf teenagers in French and in English shows unusual characteristics in the areas of the languages that can be related to space and time (e.g. Vincent-Durroux, 2009 \& 2008). Cognitive representations therefore strongly influence the deaf's first language. The discussion bears on the fact that in the context of second language acquisition by hearing subjects, the cognitive representations underlying the mother tongue could be implied in fossilisation and nativisation phenomena.

\section{INDEX}

Mots-clés : représentations cognitives, surdité, langue maternelle, langue étrangère

Keywords : cognitive representations, deafness, first language, second language

\section{AUTEUR}

\section{LAURENCE VINCENT-DURROUX}

Université Montpellier 3 / EMMA (EA 741) 